de riz se travaillait mal, donnant du beurre peu consistant et des fromages ne supportant pas la maturation. Il est intéressant de noter que ces constatations faites par les fabricants, concordent entièrement avec mes expériences et les confirment.

Me basant sur le fait connu que la composition de la graisse d'un animal peut varier selon les substances grasses qu'on lui fait absorber, j'ai pensé que l'action du son de riz était due à la matière grasse qu'il contenait et $j$ 'ai alors alimenté des vaches avec du son de riz dégraissé par pression : le beurre que j'ai obtenu présentait un index d'acides volatils solubles très bas $(18,4)$. On peut par conséquent assurer que la graisse n'a aucune influence sur l'abaissement de l'index d'acidité volatile du beurre.

Les expériences se continuent en ajoutant maintenant de la graisse de riz à l'alimentation normale, de même que hous effectuons d'autres expériences pour établir à quel autre constituant est due la dite action du son de riz et pour rechercher le moyen de la neutraliser. Dès que les expériences seront terminées, je communiquerai les résultats.

J'ai cru utile de faire connaître ces observations parce qu'elles intéressent les personnes préposées à la surveillance de la production et du commeree du lait industriel ; car, si l'action du son de riz sur les acides volatils solubles peut n'avoir aucune importance pratique en ce qui concerne le lait destiné à l'alimentation, elle en a une, au contraire, très grande en ce qui concerne le lait destiné à l'industrie, attendu que les produits que l'on obtient (beurre, fromage) du lait provenant de vaches alimentées avec du son de riz, n'offrent pas les qualités de pureté requises par la loi.

\title{
LA PASTEURISATION A TEMPÉRATURE ÉLEVÉE DE COURTE DURÉE AUX ÉTATS-UNIS
}

par

\author{
M. W. YALE
}

Section de Bactériologie de la Station d'Essais Agricoles de l'Etat de New-York, Geneva (N.-Y.).

(Suite.)

\section{REVUE BIBLIOGRAPHIQUE \\ VOLUME DE LA COUCHE DE CREME}

L'idée du consommateur, que l'épaisseur de la couche de crème dans une bouteille de lait est une indication de la richesse du lait, a amené le vendeur de lait à appliquer des méthodes qui donneront un volume maximum à la couche de crème. De telles méthodes sont 
particulièrement importantes dans le cas du lait pasteurisé, où il y a une marge étroite entre les exigences de la pasteurisation légale et le temps et la température auxquels une destruction appréciable de la montée de la crème a lieu.

Dahlberg et Marquardt (1929) et Marquardt et Dahlberg(1931) ont trouvé que 30 minutes à $144^{\circ} \mathrm{F}$. (58088 C.) et 20 secondes à $160^{\circ} \mathrm{F}$. $\left(71^{\circ} 11 \mathrm{C}\right.$.) étaient les périodes maxima de temps pendant lesquelles le lait pouvait être chambré sans réduction appréciable dans le volume de la eouche de crème. Ils utilisèrent un pasteurisateur à vaporisation Burrell pour leurs études à basse température et un petit chauffeur à tubulures internes pour leurs études à haute température. Ils ont indiqué que le temps pendant lequel le lait est à une température supérieure à $145^{\circ} \mathrm{F}$. $\left(62^{\circ} 7 \mathrm{C}\right.$.) pendant la période de chauffage, et spécialement au-dessus de $150^{\circ} \mathrm{F}$. (6505 C.), a une influence directe sur la période pendant laquelle le lait peut être maintenu à $160^{\circ} \mathrm{F} .(7101$ C.) sans danger pour la montée de la crème.

Dans les pasteurisateurs électriques et à plaques, le lait est chauffé plus rapidement, e'est-à-dire entre 10 et 20 secondes, que dans les pasteurisateurs du type à tubes intérieurs, où le chauffage dure de 100 à 150 secondes. Une plus grande latitude est, par conséquent, laissée aux pasteurisateurs électriques et à plaques en ce qui concerne les températures auxquelles le lait peut être chauffé, et en ce qui concerne la durée pendant laquelle il peut être chambré sans qu'il résulte un dommage appréciable pour la montée de la crème.

QUINN et BURGWALD (1933) ont trouvé que la pasteurisation à $143^{\circ} \mathrm{F} .\left(61^{\circ} 66\right.$ C.) pendant 30 minutes dans une cuve de chambrage nuisait moins à la montée de la crème du lait qu'une température de pasteurisation de $160^{\circ} \mathrm{F}$. ( $71^{\circ} 1 \mathrm{C}$.) pendant 15 secondes dans un pasteurisateur Isotherm. Des résultats analogues furent obtenus avec du lait pasteurisé au laboratoire. IRWIN (1933 a) commente les résultats obtenus par QUINN et BuRGWALD. Il pense que des différences dans la durée du préchauffage, la durée du chambrage, la température de l'eau de chauffage et dans le refroidissement des échantillons peuvent jouer un rôle en ce qui concerne un grand nombre des différences observées par les auteurs sur la séparation de la crème.

La synchronisation des opérations à la laiterie est plus nécessaire dans le cas de la pasteurisation à température élevée, de courte durée, spécialement avee les pasteurisateurs du type à tubes intérieurs, que dans le cas de la pasteurisation lente avec chambrage de 30 minutes, parce que les fermetures détruisent la montée de la erème du lait maintenu dans l'appareil. 


\section{SAVEUR}

Des saveurs anormales dans le lait ont pour résultat des plaintes de la part des elients sérieux, et leur prévention constitue un des problèmes les plus importants du commerçant en lait. Au moins deux de ces saveurs sont plus communes dans le lait pasteurisé que dans le lait brut. L'une est la saveur de cuit ou de surchauffé, tandis que l'autre est communément appelée saveur métallique, oxydée, suiffeuse, de carton, de papier ou de eapsule.

Le point à partir duquel la saveur de surchauffé est perceptible dans le lait pasteurisé dépend beaucoup de la température du milieu de chauffage et de la finesse de goût du consommateur. En fait, l'observateur moyen ne remarque généralement pas la saveur de surchauffé si le lait n'a pas été chauffé au-dessus de $145^{\circ} \mathrm{F}$. (6207 C.) pendant 30 minutes.

En ce qui concerne la pasteurisation haute, MARquardt et DAHLBERG (1931) ont observé que des températures de $160^{\circ} \mathrm{F}$. $\left(71^{\circ} 1\right.$ C.) ou au-dessous pendant des périodes de chambrage de 20 secondes ou moins ne donnaient pas de saveur de surchauffé au lait. Les saveurs de surchauffé ne sont pas perceptibles pour l'observateur moyen, à moins que le lait n'ait été maintenu à des températures de $160^{\circ} \mathrm{F}$. $\left(71^{\circ} 1 \mathrm{C}\right.$.) ou au-dessus pendant une période de 2 minutes ou plus.

QUINN et BURGWALD (1933) ont comparé la saveur du lait pasteurisé dans une euve de chambrage à $143^{\circ} \mathrm{F}$. (61066 C.) pendant 30 minutes avec celle d'un lait pasteurisé à $160^{\circ} \mathrm{F}$. (71011 C.) pendant 15 secondes et décidé que "la méthode à température élevée, de courte durée, donnait moins la saveur de euit que la méthode de pasteurisation lente ».

Il ne semble pas qu'on ait étudié complètement la formation comparative des saveurs métalliques ou oxydées dans le lait pasteurisé par les deux méthodes.

\section{REDUCTION DU NOMBRE DES BACTÉRIES}

Des erreurs inhérentes à la numération sur plaques d'agar standard font disparaître les différences d'efficacité des divers pasteurisateurs, à moins qu'un grand nombre de comparaisons ne soient effectuées en utilisant le même lait partagé en plusieurs parties. Aussi, de légères différences dans les numérations bactériennes sontelles sans importance, tout au moins en ce qui concerne la qualité des bouteilles de lait livrées aux consommateurs.

Pasteurisateur Electropure. - La réduction du nombre des bactéries dans les premiers modèles de pasteurisateurs Electropure fut étudiée par Anderson et FinkELstein (1919), Chilson (1921), Robison (1923), Harding (1927), les Services d'Hygiène 
Publique de l'Etat de Michigan (1922), OGDen, Carpenter et Curran (1924), les Services d'Hygiène Publique de l'Etat de Pensylvanie $(1926 b)$ et Prescott (1927). Tous ont trouvé une réduction satisfaisante par le procédé Electropure, sauf CHILsoN et HaRding. Les résultats obtenus par ces expérimentateurs ne sont pas applicables au pasteurisateur Electropure moderne, avec sa durée de chambrage plus longue et ses réglages améliorés.

Stabler (1931) a fait des essais avec un petit modèle de laboratoire du pasteurisateur Electropure et en a conclu que la pasteurisation électrique à $163^{\circ} 4 \mathrm{~F}$. $\left(73^{\circ} \mathrm{C}\right.$.) est aussi efficace, tant en ce qui concerne la réduction des numérations bactériennes qu'en ce qui concerne les sortes d'organismes détruits, que la méthode de pasteurisation lente à $143^{\circ} \mathrm{F}$. ( $61^{\circ} 66 \mathrm{C}$.) employée industriellement. La vitesse de chauffage et la durée du chambrage pour le procédé Electropure n'ont pas été indiquées.

Stark (1930) a indiqué que la méthode de pasteurisation lente du lait était plus efficace que le procédé Electropure. Cette conclusion fut basée sur le fait que le pourcentage de bactéries survivant à la pasteurisation lente allait de $1,2 \%$ à $10,6 \%$, tandis que celui de bactéries survivant au traitement par la chaleur allait de 15,0\% à $27,7 \%$ quand le procédé Electropure était utilisé. STARK a noté qu'en 10 minutes, la température de pasteurisation varia de $167^{\circ} \mathrm{F}$. $\left(75^{\circ} \mathrm{C}\right.$.) à $159^{\circ} \mathrm{F},\left(70^{\circ} 5 \mathrm{C}\right.$.). Ceci indique que le pasteurisateur étudié par lui ne fonctionnait pas convenablement à ce moment, car s'il avait fonctionné convenablement, la température aurait dû varier rapidement entre deux points déterminés d'avance, habituellement $161^{\circ}$ F. $\left(71^{\circ} 66\right.$ C. $)$ et $163^{\circ}$ F. $\left(72^{\circ} 77\right.$ C. $)$.

Pasteurisateur Precision. - Les Services d'Hygiène Publique des Etats-Unis (1930) ont observé l'effet sur des numérations totales sur plaques au cours de 10 essais effectués sur le pasteurisateur Precision. Avee une numération sur plaque effectuée sur du lait brut et donnant comme résultat de 280.000 à 910.000 bactéries par centimètre cube, le pourcentage de réduction variait de $89 \%$ à $99 \%$. Dans 5 essais avec températures moyennes à la sortie du réchauffeur de $158^{\circ} 4 \mathrm{~F}$. $\left(70^{\circ} 22 \mathrm{C}\right.$.) à $162^{\circ} 6 \mathrm{~F}$. $\left(72^{\circ} 55 \mathrm{C}\right.$.), la numération moyenne sur plaques du lait au réfrigérant était de 5.800 , comparée à une numération brute moyenne de 400.000 , ce qui représentait done une réduction moyenne de plus de $96 \%$.

Pasteurisateur Isotherm. - Les Services d'Hygiène Publique de l'Etat de New-York (HoLmQUist, 1931) ont trouvé, au cours d'une série d'essais bactériologiques effectués sur un pasteurisateur Isotherm fonctionnant journellement dans une laiterie industrielle, une réduction moyenne de plus de $96 \%$ sur la numération sur 
plaques standard. La réduction moyenne obtenue fut la même, ou même légèrement meilleure, que celle obtenue en pasteurisant dans des tubes à essai à $143^{\circ} \mathrm{F}$. $\left(61^{\circ} 66 \mathrm{C}\right.$.) pendant 30 minutes des échantillons en double du lait non traité.

Pasteurisateur à plaques York. - Les Services d'Hygiène Publique de l'Etat de Pensylvanie (1931 b) ont étudié le pasteurisateur à plaques type York pendant 4 jours d'opération. Le pourcentage de réduction moyenne obtenu sur les numérations sur plaques d'agar standard fut, pendant ces journées d'essai, de plus de $96 \%$.

Chauffage pendant 30 minutes ou chauffage très court, au laboratoire et dans des conditions industrielles. - QUINN et BURGWALD (1933) ont pasteurisé des quantités de laits séparées provenant de la même cuve dans des conditions de laboratoire et en ont conclu qu'il n'y avait pratiquement pas de différence dans l'efficacité germicide d'une température de pasteurisation de $160^{\circ} \mathrm{F}$. $\left(71^{\circ} 1 \mathrm{C}\right.$.) ou de $162^{\circ} \mathrm{F}$. $\left(72^{\circ} 2 \mathrm{C}\right.$.) pendant 15 ou 20 secondes, eomparée à une température de $143^{\circ} \mathrm{F}$. (6106 C.) pendant 30 minutes. YALE et ses collaborateurs (1933), travaillant en collaboration avec le Bureau d'Application des Lois Sanitaires au Lait des Services d'Hygiène Publique de l'Etat de New-York, ont trouvé que la pasteurisation de laboratoire à $143^{\circ} \mathrm{F}$. $\left(61^{\circ} 6 \mathrm{C}\right.$.) pendant 30 minutes donnait des numérations moins élevées que la pasteurisation de laboratoire à $160^{\circ} \mathrm{F} .\left(71^{\circ} 1 \mathrm{C}\right.$.) pendant 15 secondes dans 12 essais comparatifs sur $15(80 \%)$. La numération moyenne des échantillons pasteurisés à $143^{\circ} \mathrm{F}$. $\left(61^{\circ} 6 \mathrm{C}\right.$.) était de 6.490 par centimètre cube, alors que celle des échantillons pasteurisés à $160^{\circ} \mathrm{F}$. ( $71^{\circ 1}$ C.) était de 12.200.

QUINN et BURGWALD ont comparé aussi l'efficacité germicide sur des quantités de lait séparées provenant de la même cuve. Ce lait avait été pasteurisé industriellement dans un appareil de chambrage et dans un pasteurisateur Isotherm, Dans 6 essais comparatifs effectués sur 6 jours différents, la numération moyenne pour le lait non traité était de 1.066 .667 , de 35.087 pour le lait pasteurisé par chambrage, et de 50.271 pour le lait pasteurisé par le pasteurisateur Isotherm, tous ces chiffres par centimètre cube. La réduction moyenne pour le lait pasteurisé par chambrage fut de $96,71 \%$ et de $95,28 \%$ pour le lait pasteurisé dans le pasteurisateur Isotherm. Les auteurs conclurent que, dans des conditions industrielles, une température de $143^{\circ} \mathrm{F}$. $\left(61^{\circ} 6 \mathrm{C}\right.$.) pendant 30 minutes était légèrement plus efficace qu'une température de $160^{\circ} \mathrm{C}$. $\left(71^{\circ} 1 \mathrm{C}\right.$ C) pendant 15-20 secondes dans les essais comparatifs effectués.

En raison des différences existant dans la construction et le 
fonctionnement des pasteurisateurs, IRwIN (1933 a) indique que, tout au moins en ce qui concerne la pasteurisation industrielle, les conclusions tirées par les auteurs devraient être entre un type de pasteurisateur fonctionnant à $143^{\circ} \mathrm{F}$. $\left(61^{\circ} 6 \mathrm{C}\right.$.) pendant 30 minutes et un type de pasteurisateur rapide à température élevée fonctionnant avee diverses combinaisons de température et de durée.

Il est difficile de tirer des conclusions des études de cette nature, car, ainsi qu'Irwin (1933 a) le dit : "Les efficacités germicides de la pasteurisation à basse et à haute température sont difficiles à comparer quand on n'examine que des numérations sur agar standard. La température et le temps employés pour refroidir les échantillons ont une influence sur la croissance des bactéries, ainsi, du reste, que sur la séparation de la crème. Le temps pendant lequel les échantillons sont conservés est aussi important. L'effet que ces conditions peuvent avoir eu sur les petites différences dans les numérations sur plaques d'agar n'est pas indiqué. "

YALE et ses collaborateurs (1933); reconnaissant les difficultés indiquées par IrwIN, ont pasteurisé des quantités séparées de lait provenant de la même cuve dans des conditions industrielles avec une cuve de chambrage fonctionnant à $143^{\circ} \mathrm{F} .\left(61^{\circ} 6 \mathrm{C}\right.$.) pendant 30 minutes et avec un pasteurisateur Precision opérant à $160^{\circ} \mathrm{F}$. (7101 C.) pendant 15 secondes. La pasteurisation dans une cuve de chambrage à $143^{\circ} \mathrm{F}$. $\left(61^{\circ} 6\right.$ C.) pendant 30 minutes fournit des numérations moins élevées que la pasteurisation par le pasteurisateur Precision dans $72 \%$ des cas (13 sur 18 ). La numération moyenne du lait récemment pasteurisé à $143^{\circ} \mathrm{F}$. $\left(61^{\circ} 6 \mathrm{C}\right.$.) pendant 30 minutes était de 17.200 par centimètre eube, tandis que celle du lait récemment pasteurisé par le pasteurisateur Precision était de 20.600.

L'effet de la température et de la durée de la conservation sur la numération sur plaque standard du lait pasteurisé par le pasteurisateur Precision fut net. La numération moyenne de 31 échantillons de lait en bouteilles pasteurisé par le pasteurisateur Precision était avant conservation de 22.800 , tandis qu'après conservation à $40-45^{\circ} \mathrm{F}$. (404-702 C.) pendant 24 heures, la numération moyenne du même lait était de 9.600 , ce qui correspond à une réduction moyenne de la numération de $58 \%$. Malheureusement, la réduction de la numération pendant la conservation du lait pasteurisé dans la cuve de chambrage ne fut pas étudiée.

Conclusions. - Les différences relevées par les divers expérimentateurs ne permettent pas de conclure qu'une méthode de pasteurisation déterminée produit une bouteille de lait pasteurisé ayant une numération bactérienne plus basse que l'autre. Le fait que les installations industrielles recevant du lait brut de bonne qualité livrent facilement du lait répondant aux exigences des 
numérations bactériennes prouve que la réduction bactérienne est satisfaisante dans les deux méthodes.

\section{RÉDUCTION DES SPORES BACTÉRIENNES}

Ogden, Carpenter et Curran (1924) ont ensemencé des spores de Bacillus subtilis dans 50 gallons (226 1. 66) de lait écrémé pasteurisé et ont pasteurisé rapidement ce lait à $160^{\circ} \mathrm{F}$. $\left(71^{\circ} 1 \mathrm{C}\right.$.) avec un pasteurisateur Electropure. Des échantillons du lait ensemencé furent aussi chauffés au bain-marie à une température analogue à celle atteinte par la machine Electropure. La réduction moyenne des spores dans 2 essais fut de $79 \%$ pour le procédé Electropure, contre $85 \%$ pour la méthode au bain-marie, ce qui indique que les deux méthodes sont aussi efficaces l'une que l'autre.

GelPi et Devereux (1930) ont comparé la résistance relative des spores de Bacillus subtilis, B. mycoides, B. mesentericus et $B$. megatherium à l'Electropure et à la pasteurisation lente dans les conditions du laboratoire. Le pourcentage de réduction du nombre des spores fut de 0 à $13 \%$ dans la pasteurisation lente à $145^{\circ} \mathrm{F}$. ( $62 \circ 7$ C.) pendant 30 minutes et de 71,5 à $99,5 \%$ dans le procédé Electropure avec chambrage momentané à $160^{\circ}$ F. $\left(71^{\circ} 1\right.$ C.). Les résultats indiquaient que le procédé Electropure était plus efficace dans la destruction des endospores bactériennes que la pasteurisation lente.

Dans une publication ultérieure, GELPI et DEverkUx (1932) émettent la théorie, appuyée sur des expériences de laboratoire, que ".la destruction des endospores par ce procédé électrique n'est pas entièrement due à la chaleur créée dans le milieu entourant les endospores, mais aussi à un autre facteur de chaleur, c'est-à-dire à la chaleur engendrée à l'intérieur des endospores \%. Ainsi, ils pensent que la température atteinte à l'intérieur des endospoces est probablement plus élevée que celle du milieu environnant.

Ils n'ont pas fait d'études comparatives à des températures élevées avec de l'eau chaude comme milieu de chăuffage, ainsi que le firent OGDEN, CARPENTER et CuRRAN (1924), qui ne trouvèrent pas de différence appréciable dans les deux méthodes pour la destruction des spores.

La destruction des spores dans le lait est un côté peu important de la pasteurisation, car il y a peu de spores dans le lait frais.

\section{RÉDUGTION DES ORGANISMES DU GROUPE “ ESGHERICHIA-AEROBAGTER »}

On ne doit pas trop compter sur l'utilisation des organismes du groupe Escherichia-Aerobacter comme organismes d'essai pour déterminer l'efficacité d'un procédé de pasteurisation, en raison de la résistance variable de ses membres au traitement par la chaleur. 
La présence de ces organismes dans le lait pasteurisé en bouteilles est plus souvent due à une contamination postérieure à la pasteurisation qu'à une survie au traitement par la chaleur. La source de contamination peut être déterminée en prélevant des échantillons aux divers stades de la pasteurisation.

Pasteurisateur Electropure. - ANDERSON et FINKELSTEIN (1919) ont trouvé que le pasteurisateur Electropure détruisait réellement presque tous les organismes endopositifs fermentateurs du lactose dans le lait brut.

ChILson (1921) a trouvé Escherichia coli présent 20 fois sur 100 dans le lait sortant d'un pasteurisateur électrique industriel.

D'après OGDEN, CARPENTER et CuRRAN (1924), toutes les baetéries appartenant au groupe colon-aerogenes furent détruites par le procédé Electropure à $160^{\circ} \mathrm{F}$. $\left(71^{\circ} 1 \mathrm{C}\right.$.).

Dans des essais effectués par les Services d'Hygiène Publique de I'Etat de Pensylvanie (1926 a), du lait brut fut ensemencé avec Escherichia coli et Aerobacter aerogenes. Très peu de ces organismes passèrent à travers l'appareil Electropure à $160^{\circ}$ F. $\left(71^{\circ} 1 \mathrm{C}\right.$.). Ils furent trouvés occasionnellement après le traitement à $155^{\circ} \mathrm{F}$. $\left(68^{\circ} 33\right.$ C.) et fréquemment après le traitement à $150^{\circ} \mathrm{F}$. $\left(65^{\circ} 55 \mathrm{C}\right.$.).

Des rapports plus récents (Services d'Hygiène Publique de 1'Etat de Pensylvanie) (1926 b) et Irwin (1928) donnent le nombre d'Escherichia coli existant dans le lait traité dans deux laiteries utilisant le procédé Electropure, comparé au nombre d'Escherichia coli existant dans le lait traité dans une troisième laiterie utilisant un pasteurisateur à cuve et à serpentin horizontal. Le nombre d'Escherichia coli dépassait généralement 10.000, par centimètre cube, dans le lait non traité. Sur 127 échantillons de lait traités par le procédé Electropure, 12 seulement accusèrent la survie de Escherichia coli, tandis que sur 33 échantillons de lait traités par le pasteurisateur à cuve et à serpentin horizontal, 27 l'accusèrent. La destruction de Escherichia coli par le procédé Electropure, aussi bien dans les essais au laboratoire que dans les conditions industrielles, fut si importante, que le Comité estima possible d'employer sa destruction comme mesure de l'efficacité des appareils pasteurisateurs.

Prescote (1927) n'a pas trouvé d'Escherichia coli dans le lait traité par un pasteurisateur industriel Electropure.

Pasteurisateur Precision.- Les Services d'Hygiène Publique des Etats-Unis (1930) et des Laboratoires travaillant en collaboration avec eux ont trouvé que, pour du lait moyen, des températures de sortie de $158^{\circ} 4 \mathrm{~F}$. (7002 C.) et plus, la méthode instantanée à la vapeur réduisait une numération moyenne d'Escherichia coli 
de 5.370 par centimètre cube à une moyenne de 0 , soit une réduction de $100 \%$.

Pasteurisateur type à plaques York.- Les Services d'Hygiène Publique de l'Etat de Pensylvanie $(1931 b)$ ont trouvé que pratiquement toutes les bactéries productrices de gaz étaient détruites dans la section chauffante du pasteurisateur type à plaques York.

Pasteurisation lente d'une durée de $\mathbf{3 0}$ minutes. - Un certain nombre d'expérimentateurs différents ont étudié la survie de Escherichia coli pendant la pasteurisation lente de 30 minutes effectuée industriellement. Le travail de McCrady et LANGEvin (1932) apporte une certitude à ce sujet, en raicon du grand nombre de laiteries étudiées et du caractère sérieux de leur étude. Ces expérimentateurs canadiens ne trouvèrent, lors d'une étude de 40 laiteries employant des températures de pasteurisation de $140^{\circ} \mathrm{F}$. $\left(60^{\circ} \mathrm{C}\right.$.) à $145^{\circ} \mathrm{F}$. $\left(62^{\circ} 7 \mathrm{C}\right.$.) pendant 30 minutes, que 2 cas où les organismes du groupe coli-aerogenes étaient présents dans des quantités de $1 \mathrm{~cm}^{3}$ de lait.

Conclusions. - Dans la grande majorité des cas, la pasteurisation, qu'elle soit rapide à température-élevée, ou lente de 30 minutes de durée, détruit les organismes du groupe Escherichia-Aerobacter. Il ne semble pas y avoir de différence entre les deux méthodes en ce qui concerne cette action.

\section{DÉVELOPPEMENT DES BACTERIES THERMOPHILES}

Le développement des bactéries thermophiles dans le matériel de pasteurisation est quelquefois un problème d'importance considérable, surtout lorsque le matériel fonctionne plus de trois heures. La connaissance, que le développement des bactéries thermophiles était surtout dû à des opérations inđustrielles défectueuses, a graduellement eu pour résultat de meilleures conditions de travail, ce qui fait que la question n'est plus aussi importante qu'elle l'était à un moment donné.

BreEd (1932) a résumé les études faites sur le sujet des bactéries thermophiles dans le lait pasteurisé par pasteurisation lente en 30 minutes. Peu d'expérimentateurs ont étudié leur développement dans le procédé rapide à température élevée.

Prescott (1927) a étudié un pasteurisateur Electropure en marche ordinaire avec chauffage momentané à $158^{\circ} 5 \mathrm{~F}$. (7002 C.). La numération moyenne des bactéries thermophiles dans le lait non traité était de 20.000 par centimètre cube. Il y avait une diminution de $80 \%$ dans le lait traité. Prescott ensemença ses plaques à $50^{\circ}-55^{\circ} \mathrm{C}$., températures qui permirent la croissance de plus d'organismes que n'en auraient permis des plaques à des tempéra- 
tures plus élevées. BREED (1932) limite les termes thermophiles et aimant la chaleur aux organismes se développant rapidement à $55^{\circ} \mathrm{C}$. et considère ceci préférable à une définition abaissant la température à $50^{\circ} \mathrm{C}$.

HARDing et WARD (1930) ont noté un léger développement des bactéries thermophiles dans deux pasteurisateurs Electropuré en fonctionnement dans une laiterie en Pensylvanie. Ils utilisèrènt l'épreuve de la réduction du bleu de méthylène à $63^{\circ} \mathrm{C}$.

YaLe et KelLy (1933) ont étudié le développement des bactéries thermophiles dans 9 laiteries produisant du lait pour vente au détail, dont 4 utilisaient l'Electropure, 2 l'Isotherm, 2 le Precision et 1 le pasteurisateur à plaques York. Les numérations thermophiles furent déterminées sur plaques d'agar à l'étuve à $56^{\circ}$ ou $63^{\circ} \mathrm{C}$. et vérifiées par un examen microscopique. Dans 2 laiteries, il n'y eut pas de développement perceptible des thermophiles ; dans 5 laiteries, il y eut un léger développement; et dans 2 laiteries, un développement marqué. Les numérations maxima de thermophiles par laiterie variaient entre 40.000 et 50.000 par centimètre cube. Dans 7 des neuf laiteries, les numérations étaient inférieures à 10.000 par centimètre cube et, par conséquent, n'avaient aucune importance pratique. Le développement des thermophiles était dû à des pratiques industrielles défectueuses, plutôt qu'à un type particulier de pasteurisateur instantané.

Les numérations de thermophiles dans les neuf laiteries étudiées ne furent pas aussi élevées qu'on l'a si souvent dit à propos des laiteries utilisant la pasteurisation lente de 30 minutes à 142 $145^{\circ} \mathrm{F} .\left(61^{\circ} 1-62^{\circ} 7\right.$ C. $)$.

Dans la pasteurisation lente de 30 minutes de durée, le développement des bactéries thermophiles dans les chauffeurs régénérateurs et dans les filtres est tout à fait anaJogue à leur développement dans le procédé rapide. Toutefois, dans le pasteurisateur lent, à l'inverse de ce qui se passe dans le pasteurisateur rapide, le lait est ensuite soumis à une température voisine de l'optimum pour le développement des thermophiles. Ceci explique très bien le plus grand développement des thermophiles dans le cas du procédé lent de 30 minutes de durée.

\section{ESSAIS SUR LES BACTÉRIES PATHOGẼNES}

La température mortelle pour les bactéries pathogènes est un rapport temps-température. Ainsi, il y a de nombreuses températures mortelles différentes pour chaque espèce, chacune correspondant à un temps d'exposition déterminé. De même, de nombreuses températures différentes pouvaient être utilisées pour la pasteurisation. 
PARk (1927) déclare : "Ayant fixé la température mortelle des bactéries pathogènes dans le lait pendant la durée désirée, nous devons décider quelle chaleur additionnelle doit être appliquée au lait pendant la pasteurisation industrielle pour remédier aux défauts mécaniques et assurer ainsi un chauffage suffisant de chaque partie du lait pendant le temps voulu. C'est ce que nous appelons la marge de sécurité, et elle varie dans les différentes machines suivant la perfection de la construction et du fonetionnement. "

Depuis les années voisines de 1920, l'aptitude de la pasteurisation industrielle à détruire les bactéries pathogènes a été très étudiée par les autorités sanitaires officielles et autres. Des appareils industriels de type standard ont été employés dans ces recherches. La méthode habituelle a consisté dans l'ensemencement du lait avec un grand nombre d'organismes d'une maladie due au lait et dans l'examen ultérieur du lait traité pour vérifier la présence ou non des organismes à essayer.

Jusqu'à présent, la technique pour l'essai du matériel de pasteurisation n'a pas été codifiée et divers expérimentateurs ont utilisé des techniques différentes. Il en résulte qu'il est difficile de comparer les résultats obtenus par les divers expérimentateurs.

Heureusement, les variétés de beaucoup d'espèces de bactéries pathogènes sont pratiquement différentes dans leur résistance. NorTH et PARK (1927) déclarent : «Il n'y a pas de différence importante dans la résistance à la chaleur des variétés de bacilles de la tuberculose bovine et de la tuberculose humaine, et entre les différentes variétés de chaque espèce, ou entre les bacilles de la tuberculose dans le lait infecté naturellement, les lésions des tissus, ou les cultures pures. "

Des exceptions importantes à ce qui précède sont constituées par des variétés de streptocoques hémolytiques et des variétés d'organismes appartenant au genre Brucella, qui peuvent varier d'une façon très importante dans leur résistance à la chaleur.

Nous donnons ci-dessous les résultats de recherches sur la destruction par les pasteurisateurs industriels des organismes de la fièvre typhoïde et de la paratyphoïde, de la diphtérie, de l'angine streptococcique, de la fièvre ondulante et de la tuberculose.

\section{Organisme de la typhoïde.}

La fièvre typhoïde est une des plus importantes des maladies dues au lait, et elle est très redoutée, en raison de son taux de mortalité et de sa nature éminemment contagieuse. Heureusement, le bacille de la typhoïde est détruit à des températures relativement basses et une grande marge de sécurité est assurée par les règles actuelles de la pasteurisation. 
Pasteurisation de courte durée à température élevée. Les Services d'Hygiène Publique de l'Etat de Michigan (1922) ont trouvé, lors d'essais répétés en double, que les bacilles de la typhoïde et de la paratyphoïde étaient détruits quand le pasteurisateur Electropure fonctionnait à $149^{\circ}$ F. $\left(65^{\circ}\right.$ C. $)$, ce qui fut la plus basse température de fonetionnement.

Les Services d'Hygiène Publique de l'Etat de Pensylvanie (1926 a) ne purent pas prouver la présence de bacilles de la typhoïde dans du lait traité avec le pasteurisateur Electropure à $150^{\circ}, 155^{\circ}$ et $160^{\circ} \mathrm{F}$. $\left(65^{\circ} 5,68^{\circ} 3\right.$ et $71^{\circ} 1$ C. $)$.

Les Laboratoires des Services d'Hygiène Publique de l'Etat de New-York, de la Ville de New-York et des Etats-Unis ont collaboré à l'étude du pasteurisateur Electropure en 1927. La Section des Laboratoires et des Recherches des Services d'Hygiène Publique de l'Etat de New-York ne put prouver la présence des bacilles de la typhoïde dans le produit final de tous les prélèvements effectués. Ceux-ci comprenaient des prélèvements effectués sur des opérations à des températures moyennes de $1.54^{\circ} 8 \mathrm{~F}$. $\left(68^{\circ} 2 \mathrm{C}\right.$.) à $163^{\circ} 2 \mathrm{~F}$. ( $72^{\circ} 8$ C.). Le Laboratoire de la Ville de New-York a isolé des bacilles typhiques dans du lait chauffé à $140^{\circ} \mathrm{F}$. $\left(60^{\circ} \mathrm{C}\right.$.) ; mais tous les autres prélèvements furent négatifs. Les Services d'Hygiène Publique des Etats-Unis ne firent pas d'essais de laboratoire avec ces organismes.

Pasteurisation lente de $\mathbf{3 0}$ minutes. - Des essais sévères de divers types de pasteurisateurs lents furent effectués par NoRTH et ses collaborateurs (1925) dans les expériences d'Endicott. Des pasteurisateurs lents des types à courant, à cuve et à poche, furent étudiés. Dans une série d'essais, les résultats avec les types de machines à courant et à cuve furent positifs après 30 minutes de chamb rage à $142^{\circ} \mathrm{F}$. (6101 C.), ce qui est dû, pense-t-on, à des défauts dans le type d'appareil pasteurisateur indiqué par les ingénieurs. Dans une série d'essais postérieure, tous les résultats furent négatifs à $142^{\circ} \mathrm{F} .\left(61^{\circ} 1 \mathrm{C}\right.$.), ce qui est dû, pense-t-on, à une amélioration dans le type d'appareil pasteurisateur et dans les méthodes de fonctionnement.

Conclusions. - Les résultats obtenus dans ces recherches indiquent que les deux méthodes de pasteurisation sont efficaces pour détruire les organismes de la fièvre typhoïde quand les pasteurisa teurs fonctionnent convenablement.

\section{Organisme de la diphtérie.}

Les températures mortelles de l'organisme de la diphtérie sont parmi les plus basses de celles de toutes les espèces pathogènes trouvées dans le lait. Pour cette raison, la destruction de cet orga- 
nisme n'est pas un indice satisfaisant de la sécurité du lait traité, et il n'a pas été employé sur une grande échelle comme organisme d'essai pour déterminer l'efficacité du matériel pasteurisateur.

Pasteurisation de courte durée à température élevée. Les études de la destruction de l'organisme de la diphtérie par la pasteurisation de courte durée à température élevée n'ont été effectuées qu'avec le pasteurisateur Electropure.

Les Services d'Hygiène Publique de l'Etat de Michigan (1922) ont, lors d'expériences répétées en double, trouvé que les organismes de la diphtérie étaient détruits quand le pasteurisateur Electropure fonctionnait à $149^{\circ} \mathrm{F}$. $\left(65^{\circ} \mathrm{C}\right.$.). Cette température fut la plus basse température à laquelle l'appareil fonctionna.

Des résultats analogues furent obtenus par les Services d'Hygiène Publique de l'Etat de Pensylvanie (1926 a). Ils ne permirent pas de déceler d'organismes de la diphtérie dans du lait traité avec le pasteurisateur Electropure à $150^{\circ} \mathrm{F}$. $\left(65^{\circ} 5 \mathrm{C}\right.$.) à $155^{\circ} \mathrm{F} .\left(68^{\circ} 3 \mathrm{C}\right.$.) et à $160^{\circ} \mathrm{F} .\left(71^{\circ} 1 \mathrm{C}\right.$.).

Pasteurisation lente d'une durée de 30 minutes. - Dans les expériences d'Endicott (NoRTH et ses collaborateurs, 1925), des bacilles de la diphtérie furent détruits par les pasteurisateurs des types à eourant et à poche pasteurisant en 30 minutes. On n'essaya pas d'autres types de pasteurisateurs. Les organismes furent détruits par ehauffage momentané à $129^{\circ} \mathrm{F}$. (53088 C.).

Conclusions. - Les recherches ci-dessus indiquées indiquent que les deux méthodes de pasteurisation sont susceptibles de détruire les organismes de la diphtérie avec une grande marge de sécurité.

\section{Streptocoques hémolytiques.}

Pasteurisation de courte durée à température élevée. Les Services d'Hygiène Publique de l'Etat de Michigan (1922) ont trouvé, lors d'expériences effectuées en double, que les streptocoques hémolytiques étaient détruits quand le pasteurisateur Electropure fonctionnait à $149^{\circ} \mathrm{F}$. $\left(65^{\circ} \mathrm{C}\right.$.), ee qui fut la plus basse température de fonctionnement de l'appareil.

OGDEN, Carpenter et CurRan (1924) ont trouvé qu'aucun des cobayes inoculés avec $2 \mathrm{~cm}^{3}$ de lait pasteurisé par le procédé Electropure à $160^{\circ} \mathrm{F}$. $\left(65^{\circ} 5 \mathrm{C}\right.$.), $155^{\circ} \mathrm{F}$. $\left(68^{\circ} 3 \mathrm{C}\right.$.) et $150^{\circ} \mathrm{F}$. (7101 C.) ne mourait de péritonite, tandis que beaucoup sont morts d'une péritonite dont des streptocoques bêta-hémolytiques ont été obtenus, lorsque $2 \mathrm{~cm}^{3}$ de lait non traité (lait brut de mauvaise qualité, contenant beaucoup de streptocoques alpha et bêta-hémolytiques) avaient été inoculés intra-péritonéalement aux cobayes. 
Les Services d'Hygiène Publique de l'Etat de Pensylvanie (1926 a) ont inoculé à doses massives du lait avec des cultures d'un streptocoque hémolytique. La présence de streptocoques hémolytiques ne fut révélée dans aucun des échantillons de lait traités à $150^{\circ}$ F. $\left(65^{\circ} 5\right.$ C. $), 155^{\circ}$ F. $\left(68^{\circ} 3\right.$ C. $)$ et $160^{\circ}$ F. ( $71^{\circ} 1$ C. $)$.

Le Laboratoire de Recherches de la Ville de New-York (Services d'Hygiène Publique de l'Etat de New-York, 1928) a procédé à des expériences sur l'aptitude du pasteurisateur Electropure à détruire les streptocoques hémolytiques isolés de cas de fièvre scarlatine et d'érésipèle. Les essais ont montré qu'environ un streptocoque par 10 millions passait à travers l'appareil pasteurisateur quand celui-ci fonctionnait à une température moyenne d'environ $140^{\circ} \mathrm{F}$. $\left(60^{\circ} \mathrm{C}\right.$.), Les essais ne furent pas pratiqués à des températures plus élevées, de sorte que l'aptitude du pasteurisateur Electropure à détruire des streptocoques hémolytiques dans les conditions normales de travail n'a pas été déterminée. Les Services d'Hygiène Publique de I'Etat de Pensylvanie $(1931$ b) ont ensemencé du lait avee des streptocoques hémolytiques provenant de cas d'angine et de fièvre scarlatine. Le lait ensemencé fut passé par un pasteurisateur à plaques du type York et traité à environ $160^{\circ} \mathrm{F}$. (7101 C.) pendant 15 secondes. 12 des 16 échantillons de lait préchauffé prélevés à l'entrée du chambreur furent positifs, tandis qu'aucun des 16 échantillons du lait pasteurisé prélevés à la sortie de la plaque chambreuse n'a été trouvé positif. Ces résultats ont indiqué qu'une durée de chambrage déterminée était nécessaire pour détruire ces organismes.

Pasteurisation lente de 30 minutes de durée. - PARK (1927), étudiant les températures mortelles trouvées par divers expérimentateurs pour les streptocoques hémolytiques, déclare: "On peut probablement dire avec certitude qu'une température de $138^{\circ} \mathrm{F}$. (5808 C.) pendant 30 minutes détruira tous les streptocoques hémolytiques du lait transmettant la maladie à l'homme, c'està-dire ceux de l'angine streptococcique, de la fièvre scarlatine, de l'amygdalite et des autres infections streptococciques rares. "

Dans les expériences d'Endicott (NorTH et collaborateurs, 1925), des streptocoques hémolytiques furent détruits dans les pasteurisateurs lents (30 minutes) de types à courant et à poche. On n'expérimenta aucun autre type de pasteurisateur. Les organismes furent détruits par ehauffage momentané à $129^{\circ} \mathrm{F}$. (5308 C.).

Conclusions. - Il est évident que les deux méthodes de pasteurisation détruisent tous les streptocoques hémolytiques du lait transmettant la maladie à l'homme quand de tels pasteurisateurs fonctionnent convenablement. 


\section{Organismes du genre « Brucella ».}

La destruction des organismes du genre Brucella par la pasteurisation est indispensable, en raison de leur rapport avec la fièvre. ondulante chez l'homme. Ce n'est que dans les toutes dernières années que l'importance du problème a été reconnue. Des variations dans la résistance à la chaleur des diverses espèces d'organismes de la fièvre ondulante ont rendu difficile l'établissement de températures mortelles précises.

Pasteurisation rapide à haute température. - CARPENTER et HUDDLESON (1925) ont étudié l'efficacité du procédé Electropure pour la destruction d'organismes appartenant au genre Brucella. Du lait brut fut ensemencé en doses massives avec une culture isolée d'un malade souffrant de fièvre ondulante. Aucun développement des organismes ne fut constaté dans le lait traité à $155^{\circ} \mathrm{F}$. $\left(68^{\circ} 3 \mathrm{C}\right.$.) et à $160^{\circ} \mathrm{F} .\left(71^{\circ} 1 \mathrm{C}\right.$.), tandis qu'il en fut constaté un considérable dans le lait non traité.

D'autres études du procédé Electropure ont été effectuées à l'Institut de Technologie du Massachusetts, par des représentants de l'Institut de Technologie du Massachusetts, des représentants de l'Ecole Supérieure de l'Etat de Michigan et des représentants de l'Université de Cornell (Prescots et collaborateurs, 1926). Chacun des trois groupes examina indépendamment des échantillons du lait traité. Du lait brut destiné à la vente au détail fut ensemencé en doses massives avec des organismes de Brucella composés d'une variété isolée d'un fœetus bovin avorté, d'une variété isolée d'un cas. humain de fièvre ondulante, et d'une variété isolée du sang d'un cas humain de fièvre de Malte.

Avec l'appareil Electropure fonctionnant à $150^{\circ} \mathrm{F} .\left(65^{\circ} 5 \mathrm{C}\right.$.), 11 des 12 cobayes inoculés avec le lait traité eurent des lésions typiques, ce qui indique que les organismes Brucella n'étaient pas réellement détruits.

A $155^{\circ}$ F. $\left(68^{\circ} 3\right.$ C. $), 3$ cobayes sur 13 en eurent de typiques, prouvant par là que des organismes Brucella viables résistaient au traitement par la chaleur.

A $160^{\circ} \mathrm{F} .\left(71^{\circ} 1 \mathrm{C}\right.$.), aucun des 12 cobayes inoculés n'en eut de typiques, ce qui prouve que les organismes Brucella étaient réellement détruits à la température habituellement employée dans la pratique industrielle.

Pasteurisation lente de $\mathbf{3 0}$ minutes de durée. - La plupart: des expérimentateurs ayant travaillé au laboratoire ont trouvé que tous les organismes étaient détruits à $140^{\circ} \mathrm{F} .\left(60^{\circ} \mathrm{C}\right.$.) pendant $15 \mathrm{mi}$ nutes et en ont conclu que la pasteurisation industrielle à $142^{\circ} \mathrm{F}$. 
pendant 30 minutes serait suffisante pour rendretous ces organismes non viables.

Plusieurs expérimentateurs ont étudié les pasteurisateurs industriels. Sмптн (1932) ne put obtenir Brucella abortus sur 187 échantillons traités par pasteurisation lente de 30 minutes à $145^{\circ} \mathrm{F}$. (62077 C.). Murray, MoNutw et Purwin (1932) ont trouvé que les pasteurisateurs à cuve ne sont pas parfaits, à moins qu'ils ne fonetionnent bien. Des espèces bovine et porcine survivaient à des pasteurisations de 30 minutes à $140^{\circ} \mathrm{F}$. ou $145^{\circ} \mathrm{F}$. $\left(60^{\circ}\right.$ ou $62^{\circ} 7^{\mathrm{C}}$.) quand le couvercle du pasteurisateur était laissé ouvert et quand le robinet de sortie n'était pas nettoyé.

Conclusions. - Les résultats des études effectuées au laboratoire et dans des conditions industrielles indiquent que les organismes du genre Brucella sont détruits par les deux méthodes de pasteurisation quand les pasteurisateurs fonctionnent convenablement.

(A suivre.)

\section{REVUE}

\section{LA FABRICATION DU SUCRE DE LAIT}

\section{par G. GENIN}

Les compagnies laitières, ainsi que les fabriques de caséine, ont à leur disposition de grandes quantités de lait écrémé, qui sont susceptibles de constituer une source intéressante de lactose et dont l'utilisation correspond à un revenu additionnel pour cette industrie.

Le lait de vache renferme en solution vraie environ $4,7 \%$ de lactose. Lorsque, par fermentation, sa richesse en aeide lactique s'élève à $0,6 \%$, il coagule spontanément, mais le lactose que l'on obtient dans ces conditions, par eoncentration du sérum, n'est pas cristallin. On n'obtient des eristaux parfaitement formés, correspondant à Ia formule $\mathrm{C}^{12} \mathrm{H}^{22} \mathrm{O}^{11}+\mathrm{H}^{2} \mathrm{O}$, qu'en effectuant la eristallisation du lactose en partant de ses solutions aqueuses pures. Si les cristaux de lactose sont chauffés à $110-130^{\circ}$, ils perdent leur eau de cristallisation, et si on élève encore la température, le produit devient jaune, puis brun, et donne naissance à du lacto-caramel.

La préparation du lactose constitue donc une industrie tout à fait particulière, dont les caractéristiques ont été étudiées récemment dans la revue anglaise The Chemical Trade Journal, et nous avons emprunté à cette étude les renseignements qui suivent.

\section{L'influence de la fermentation lactique.}

Le lactose est insoluble dans l'alcool et dans l'éther, mais se 